\title{
Intestinal endometriosis
}

\author{
Ayman Al-Talib • Togas Tulandi
}

Received: 7 July 2009 /Accepted: 21 August 2009/Published online: 15 September 2009

(C) Springer-Verlag 2009

\begin{abstract}
A 46-year old woman presented with chronic and intractable pelvic pain persisting for several months. She claimed to have no symptoms related to bowel or urinary function.
\end{abstract}

\section{Keywords Endometriosis $\cdot$ Pelvic pain}

A 46-year old woman presented with chronic and intractable pelvic pain persisting for several months. She claimed to have no symptoms related to bowel or urinary function. Her past history revealed a caesarean delivery and a laparoscopic removal of an ovarian dermoid cyst. A transvaginal ultrasound examination of the pelvis showed a posterior intramural fibroid of $46 \mathrm{~mm}$ in diameter and left ovarian cyst of $35 \mathrm{~mm}$ in diameter consistent with endometrioma.

The patient underwent laparoscopic total hysterectomy and bilateral salpingo-oophorectomy (BSO). Besides the presence of uterine myomata and ovarian endometrioma, we found bluish black cystic lesions on the ileum that we excised (Fig. 1). These typical endometriosis lesions involved the serosa and encompassed the muscularis of the bowel with no further penetration into the intestinal wall. The operation and postoperative course were uneventful. The chronic pain that she experienced before surgery subsided. Histopathological examination confirmed the operative findings.

Endometriosis is defined as the presence of endometrial glands and stroma outside the endometrial cavity. The implants are usually located in the pelvis, but they can be

A. Al-Talib $\cdot$ T. Tulandi $(\bowtie)$

Department of Obstetrics and Gynecology, McGill University, Montreal, Canada

e-mail: togas.tulandi@mcgill.ca found elsewhere in the body. The diagnosis is established by visualization either by laparoscopy or laparotomy [1-4].

In a meta-analysis of 7,000 women with endometriosis, the prevalence of intestinal endometriosis is $12 \%$ [5]. Depending on the vigilance and thoroughness of the examination, it varies between $3 \%$ to $37 \%$ [5-7]. The most common site of intestinal endometriosis is at the rectosigmoid area (72\%) [8]. This is followed by the rectovaginal septum (13\%), small intestine $(7 \%)$, cecum $(3.6 \%)$, and appendix (3\%) [5]. For lesions in the rectum, rectal ultrasound can determine the depth of intestinal infiltration. In one study, endometriosis with muscular infiltration was encountered in $71 \%$ of cases and serosal infiltration in $9.6 \%$ [9]. Penetration into the intestinal lumen was found in $4.8 \%$ of cases [9].

Intestinal endometriosis is usually asymptomatic and found incidentally during surgery for other conditions.

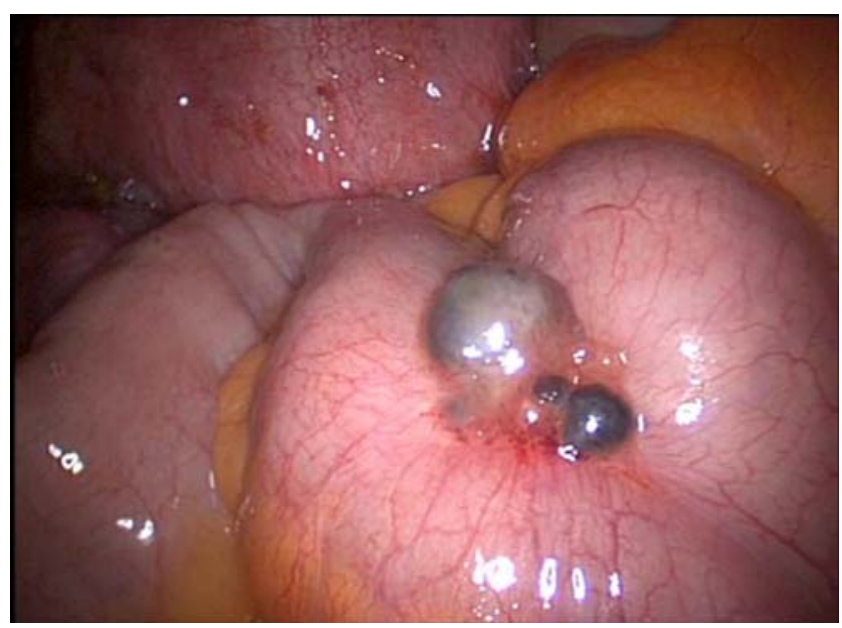

Fig. 1 Laparoscopic appearance of endometriosis lesions on the small intestine 
Under the influence of estrogen, endometriosis implants may proliferate, forming endometriosis nodule or cyst (endometrioma), and rarely infiltrate the bowel lumen.

Endometriosis implants in the lumen of the sigmoid or rectum are associated with catamenial rectal bleeding or dyschezia. However, their presence in the small intestine is often asymptomatic. Nonspecific symptoms include nausea, vomiting, abdominal swelling, diarrhea, constipation, and abdominal pain. These symptoms mimic other intestinal disorders such as irritable bowel syndrome, Crohn's disease, ulcerative colitis, and bowel cancer. Occasionally, stricture of the small bowel due to fibrosis of the endometriosis in the muscularis and serosa of the intestine leads to bowel obstruction and perforation $[10,11]$.

Treatment of intestinal endometriosis depends on the clinical findings. Resection of the affected intestine is needed for endometriosis-related bowel obstruction, and $60 \%$ to $100 \%$ of patients reported an improvement in endometriosis-related pain after excision of deeply infiltrating lesions [4]. Nonpenetrating lesion could be excised followed by estrogen suppression in the form of oral contraception, progesterone, or gonadotropin releasing hormone agonist (GnRHa). The latter is associated with initial estrogen stimulation in the first week of the treatment followed by suppression. The initial estrogen increase might stimulate proliferation of endometriosis. In women whose intestinal endometriosis has not been excised, GnRHa might lead to bowel obstruction and perforation $[10,11]$. Due to high progesterone level in pregnancy, the best treatment for women wishing to conceive is pregnancy.

Aromatase inhibitors have also been advocated as a treatment for endometriosis [12]. Its efficacy for the treatment of intestinal endometriosis is unknown. Since endometriosis is estrogen-dependent, the definitive treatment of endometriosis in general is removal of both ovaries. In addition to BSO, we performed excision of the intestinal lesions. The adequacy of BSO only remains unclear. The disease could recur, especially in those treated with cyclic hormonal replacement treatment [13]. In addition, one should rule out the presence of malignancy [14]. Women with severe or intestinal endometriosis are better treated in a center with expertise in this field.
Conflict of interest There is no actual or potential conflict of interest in relation to this article.

\section{References}

1. The Practice Committee of the American Society for Reproductive Medicine (2006) Treatment of pelvic pain associated with endometriosis. Fertil Steril 86:S18-S27

2. Kennedy S, Bergqvist A, Chapron C, behalf of the ESHRE Special Interest Group for Endometriosis and Endometrium Guideline Development Group et al (2005) ESHRE guideline for the diagnosis and treatment of endometriosis. Hum Reprod 20 (10):2698-2704

3. Link B (2006) Endometriosis and infertility. Fertil Steril 86(4): S156-S160

4. CNGOF Guidelines for the Management of Endometriosis "Collège National des Gynécologues et Obstétriciens Français ", 2006. http://www.cngof.asso.fr/D_TELE/RPC_endometriose en BM.pdf

5. Macafee CH, Greer HL (1960) Intestinal endometriosis. A report of 29 cases and a survey of the literature. J Obstet Gynaecol Br Emp 67:539-555

6. Redwine D (2003) Evidence for asymmetric distribution of sciatic nerve endometriosis. Obstet Gynecol 102(6):1416-1417

7. Remorgida V, Ferrero S, Fulcheri E et al (2007) Bowel endometriosis: presentation, diagnosis, and treatment. Obstet Gynecol Survey 62(7):461-470

8. Ribeiro PA, Rodrigues FC, Kehdi IP et al (2006) Laparoscopic resection of intestinal endometriosis: a 5-year experience. J Minim Invasive Gynecol 13(5):442-446

9. Ribeiro HSA, Aoki T (2007) 239: double-contrast barium enema and transrectal endoscopic ultrasonography in the diagnosis of intestinal deeply infiltrating endometriosis. J Minim Invasive Gynecol 14(6S):87

10. Preziosi G, Cristaldi M, Angelini L (2007) Intestinal obstruction secondary to endometriosis: a rare case of synchronous bowel localization. Surg Oncol 16:161-163

11. Ledley GS, Shenk IM, Heit HA (1988) Sigmoid colon perforation due to endometriosis not associated with pregnancy. Am J Gastroenterol 83(12):1424-1426

12. Ferrero S, Abbamonte LH, Anserini $P$ et al (2005) Future perspectives in the medical treatment of endometriosis. Obstet Gynecol Surv 60(12):817-826

13. Matorras R, Elorriaga MA, Pijoan JI et al (2002) Recurrence of endometriosis in women with bilateral adnexectomy (with or without total hysterectomy) who received hormone replacement therapy. Fertil Steril 77(2):303-308

14. Kelly P, McCluggage WG, Gardiner KR, Loughrey MB (2008) Intestinal endometriosis morphologically mimicking colonic adenocarcinoma. Histopathology 52(4):510-514 